\title{
7 ESCALA DE ENVOLVIMENTO COM O ÁLCOOL PARA ADOLESCENTES (AAIS): ANÁLISE FACTORIAL CONFIRMATÓRIA
}

\author{
| Manuela Ferreira1; Gonçalo Valente²; Lídia Cabral³; João Duarte; Amadeu Gonçalves ${ }^{5}$; Joana Andrade ${ }^{6}$ |
}

\section{RESUMO}

CONTEXTO: O consumo de álcool na adolescência contribui significativamente para produzir danos físicos e psicológicos e está associado a intoxicações, comportamentos sexuais de risco e tentativas de suicídio. A validação de uma escala que permita avaliar o envolvimento com o álcool para adolescentes é de importância capital para o planeamento de ações promotoras de estilos de vida saudáveis junto deste grupo etário OBJECTIVO: Estudar a validade e fidedignidade da Escala de Envolvimento com o Álcool para Adolescentes

MÉTODO: Recorreu-se a um modelo de investigação quantitativo, transversal, descritiva e correlacional. Participaram 971 estudantes do ensino secundário público e cooperativo. O Instrumento de recolha de dados inclui o questionário sociodemográfico, a Escala de Envolvimento com o Álcool para Adolescentes de Mayer \& Filstead (1979) adaptada por Fonte \& Alves (1999).

RESULTADOS: Os estudantes com idades compreendidas entre os $14-21$ anos, são na sua maioria rapazes (50,80\%), Revelaram-se bebedores habituais sem problemas (75,30\%), com elevadas expectativas face ao álcool (45,10\%).

Procedemos ao estudo de fiabilidade e validade da Escala de Envolvimento com o Álcool para Adolescentes e após análise factorial de componentes principais, com rotação ortogonal de tipo varimax, obtivemos 2 factores com raízes lactentes superiores a 1, que no seu conjunto explicaram $55,41 \%$ da variância total. O Alfa de Cronbach para a globalidade da escala foi de $\alpha=0.900$, com um coeficiente de Split-half para a primeira metade $=0,865$ e segunda metade $=0,800$

CONCLUSÃO: As propriedades psicométricas da escala de envolvimento com o álcool para adolescentes (AAIS) certificam a sua qualidade, enquanto ferramenta a utilizar por profissionais de saúde para avaliação dos comportamentos e sentimentos dos asolescentes face ao alcool

\section{PALAVRAS-CHAVE: Adolescentes, Álcool, Escala, Expectativas}

\section{RESUMEN}

\section{"Escala Implicación con Alcohol para Adolescentes (AAIS): análisis factorial confirmatorio"}

CONTEXTO: El consumo de alcohol en la adolescencia contribuye significativanebte a daños físicos y psicológicos, y se asocia con intoxicaciones, el comportamiento sexual de riesgo y los intentos de suicidio. La validación de una escala para evaluar la implicación con alcohol a los adolescentes es de capital importancia para la planificación de las acciones de promoción de estilos de vida saludables entre este grupo de edad.

OBJETIVO: Estudiar la validez y fiabilidad de la Escala de Participación con Alcohol a los Adolescentes.

METODOLOGÍA: Recurrido a un modelo de investigación cuantitativa, transversal, analítico, descriptivo y correlacional. Asistieron 971 estudiantes de la escuela pública y cooperativa. El protocolo de evaluación incluye el cuestionario sociodemográfico, la escala de implicación con alcohol a los adolescentes Mayer \& Filstead (1979) adaptado por Fonte \& Alves (1999).

RESULTADOS: Los estudiantes mayores de 14 años - 21, la mayoría de los hombres $(50,80 \%)$, se dieron a conocer los bebedores normales sin problemas $(75.30 \%)$ con altas expectativas hacia el alcohol (45,10\%). Procederemos Escala de validación Implicación con alcohol para adolescentes y después de un análisis factorial de componentes principales con rotación ortogonal varimax tipo, obtenido dos factores con los infantes raíces superiores a 1 , lo que en conjunto explican el $55,41 \%$ de la varianza total. El alfa de Cronbach para la escala entera fue $\alpha=0,900$, con un coeficiente de división por mitades primera mitad $=0,865,0,800$ segundos $=$ la mitad. CONCLUSIONES: Propiedades psicométricas dela escala de participación con alcohol para los adolescentes (AAI) certificar su calidad como una herramienta para ser utilizado por los profesionales de la salud para evaluar el comportamiento y los sentimientos de adolescentes enfrentan al alcohol.

\section{DESCRIPTORES: Adolescentes, Expectativas, Escala, Alcohol}

\begin{abstract}
“Teen Engagement Scale (AAIS): Confirmatory Factor Analysis”

BACKGROUND: Alcohol consumption during adolescence contributes significantly to physical and psychological harm and is associated with intoxication, sexual risk behavior and suicide attempts. Validating a scale that allows the evaluation of adolescents' involvement with alcohol is of capital importance in order to plan promoting healthy lifestyles actions. AIM: To validate the Scale of adolescents' involvement with alcohol. METHODS: We resorted to a quantitative, cross-sectional, analytical, descriptive and correlational research model with a sample of 971 high school students from public and cooperative schools. The evaluation protocol includes a sociodemographic questionnaire, the Scale Adolescents' Involvement with Alcohol of Mayer \& Filstead (1979) adapted by Fontes \& Alves (1999).

RESULTS: Students aged between 14 and 21 years old, mostly boys (50.80\%). They were habitual drinkers without problems $(75.30 \%)$, with high expectations regarding alcohol (45.10\%). We performed the study of reliability and validity for the Alcohol Involvement Scale for Adolescents. After factorial analysis of the main components with orthogonal rotation of the varimax type, we obtained 2 factors with suckling roots higher than 1 , which together accounted for $55.41 \%$ of the total variance. The Cronbach's alpha for the whole scale was $\alpha=0.900$, with a split-half coefficient for the first half $=0.865$ and for the second half $=0.800$.

CONCLUSIONS: The psychometric properties of the alcohol involvement scale for adolescents (AISA) certify its quality, as a tool to be used by health professionals to assess adolescents' behaviors and feelings regarding alcohol.
\end{abstract}

\section{KEYWORDS: Adolescents, Alcohol, Scale, Expectations}

Submetido em 30-01-2017

Aceite em 30-06-2017

\footnotetext{
1 Doutora; Professora coordenadora; Instituto Politécnico de Viseu, Escola Superior de Saúde, CI\&DETS, Portugal, mmcferreira@gmail.com

2 Mestre; Enfermeiro, Centro Hospitalar Universitário de Coimbra, Portugal, goncalo1043@hotmail.com

3 Doutora; professora coordenadora; Instituto Politécnico de Viseu, Escola Superior de Saúde, CI\&DETS, Portugal, lcabral@essv.ipv.pt

4 Doutor; Professora coordenador, Instituto Politécnico de Viseu, Escola Superior de Saúde, CI\&DETS, Portugal, duarte.johnny@gmail.com

5 Doutor; Professora adjunto, Instituto Politécnico de Viseu, Escola Superior de Saúde, CI\&DETS, Portugal, agoncalvessv@hotmail.com

6 Mestre, Médica, Centro Hospitalar Tondela Viseu, Portugal, joana.andrade99@gmail.com

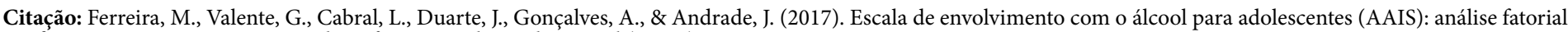
confirmatória. Revista Portuguesa de Enfermagem de Saúde Mental (Spe. 5), 39-43.
} 


\section{INTRODUÇÃO}

O envolvimento dos adolescentes com o álcool pode assumir duas formas: o situacional e o duradouro. Se o adolescente apresenta já um grande envolvimento com o consumo de bebidas alcoólicas, deixando-se assim, "absorver profundamente nesta actividade", tal como refere Barros (2003), é urgente intervir de forma a reduzir e/ou eliminar este comportamento de risco, tão nocivo para a sua saúde.

Portugal é dos países-membros da União Europeia com um dos maiores consumos de bebidas alcoólicas e de prevalência de problemas ligados ao álcool, associando-se nos últimos anos, à política geral europeia de controlo dos mesmos para uma melhor saúde do indivíduo e da comunidade (Mello, Barrias \& Breda, 2001). O aumento do alcoolismo juvenil é uma realidade, para a qual é necessário encontrar soluções. As consequências negativas deste tipo de consumo são elevadíssimas e têm conduzido a uma crescente preocupação, não só da Organização Mundial de Saúde, mas de vários organismos da União Europeia. A longo prazo e de uma forma genérica, o consumo nocivo de álcool acarreta consequências a nível fisiológico, nomeadamente ao nível do sistema metabólico e endócrino, gastrointestinal, nervoso e cardiovascular.

Nas últimas décadas desenvolveram-se vários instrumentos de auto-avaliação com o objectivo de proporcionarem uma detecção precoce do consumo abusivo de álcool e a determinação da severidade dos problemas com ele relacionado. Considerando o valor do seu uso em contexto clinico a Escala de Envolvimento com o Álcool para Adolescentes desenvolvida por Mayer \& Filstead em 1979, adaptada para adolescentes portugueses por Fonte \& Alves (1999) permite quantificar o grau de envolvimento dos adolescentes com o uso de álcool. Assim foi nosso objectivo estudar a validade e fidedignidade da Escala de Envolvimento com o Álcool para Adolescentes

\section{METODOLOGIA}

A escala de envolvimento com o álcool para adolescentes (AAIS), desenvolvido por Mayer e Filstead é um questionário de auto-avaliação constituído por 14 questões, dirigido especificamente para populações jovens (Reis et al., 2011), com o objectivo de quantificar o grau de relacionamento destes com o uso do álcool. Foi adaptado para a população adolescente portuguesa por Fonte \& Alves (1999).
Os itens versam questões como a frequência, data da ingestão da última bebida alcoólica, motivos para beber, tipo de bebida consumida habitualmente, data e com quem iniciaram o consumo, horas do dia em que costumam beber, quantidade, tipos de companhia, efeitos produzidos pelo álcool, opinião do próprio e dos outros sobre si mesmos (Fonte \& Alves, 1999).

A pontuação total varia de 0 (zero) a 79 pontos. A cada alínea "a)" corresponde um ponto (excepto nas questões 1, 2, 6, 12, 13 e 14, em que corresponde a zero pontos), cada alínea "b)" dois pontos e assim sucessivamente. Cada questão tem entre 4 a 8 respostas de escolha múltipla, de modo a permitir que o inquirido seleccione a que melhor corresponde à sua situação. Em cada uma das questões pode ser seleccionada mais do que uma alínea, contudo, para efeitos de pontuação, apenas é contabilizada a resposta de maior pontuação. Uma questão não respondida pontua-se com 0 (zero) pontos. Os resultados encontramse agrupados em 5 categorias: abstinentes ( 0 pontos); bebedores irregulares (1-19 pontos); bebedores habituais sem problemas (20-41 pontos); bebedores habituais com problemas (42-57 pontos) e "alcoholic like" (58-79 pontos) (Fonte \& Alves, 1999). Todos os itens da Escala têm um índice de discriminação elevado $(>0,71)$ e só um item apresenta uma correlação com pontuação total inferior a $r=0,43$. Medidas de fiabilidade do questionário apresentam um coeficiente de alfa de cronbach de 0,887 . Quanto à análise factorial, na sua validação inicial revela-se uma estrutura constituída por três factores, responsáveis por $58 \%$ da variância total (41,9\% para o factor $1,9,2 \%$ para o factor 2 e $7,1 \%$ para o factor 3) (Fonte \& Alves, 1999).

A escala foi aplicada numa amostra constituída por 971 alunos do ensino secundário $\left(10^{\circ}, 11^{\circ}\right.$ e $12^{\circ}$ anos) que frequentavam o mesmo estabelecimento de ensino. Foram efectuados estudos de fiabilidade (consistência interna e homogeneidade dos itens (Duarte, 2008) com determinação do coeficiente de correlação de Pearson, determinação do coeficiente alfa de cronbache e correlação de split-half.

\section{Resultados psicométricos da Escala de Envolvim- ento com o Álcool em Adolescentes (AAIS)}

Iniciou-se a avaliação das propriedades psicométricas da escala de envolvimento com o álcool em adolescentes através dos estudos de fiabilidade e de validade. Para o estudo da consistência interna determinamos: 
(i) o coeficiente de correlação de Pearson das diversas questões com o valor global; (ii) o coeficiente alfa de cronbach, o qual mede a correlação entre cada item da escala com os restantes que, supostamente, pertencem a um mesmo universo. Os parâmetros variam entre zero (0) e (1), sendo a consistência interna considerada muito boa a partir de 0,8 ; (iii) correlação de split-half ou método das metades, a qual constitui uma forma de comprovar se uma das metades dos itens da escala se revela tão consistente a medir o constructo como a outra metade (Duarte, 2008).

Quanto aos estudos de validade consideramos a validade constructo por ser a mais importante.

Recorreu-se à análise factorial dos itens e dos resultados. Usando-se o método dos componentes principais (Pestana \& Gageiro, 2008).

Na rotação dos factores foi utilizada a rotação ortogonal do tipo varimax por ser a mais recorrente e porque mantém a independência dos factores. Por este processo, os itens que incorporam cada factor são os que se correlacionam mais fortemente com cada um deles (Pestana \& Gageiro, 2008).

Como critérios de determinação do número correcto de factores a serem extraídos, considerou-se o critério de auto-valor (eigenvalue) superior a 1 e o teste de scree plot de Cattel. Na retenção dos itens cargas factoriais iguais ou superiores a 0,40 .

Ainda relativamente à análise factorial, alguns autores defendem que no sentido de haver precisão dos factores dela resultante deverá ser tida em consideração a dimensão da amostra. Embora não sendo consensual, Duarte (2008) refere que o "N" da amostra deverá ser no mínimo igual a cinco vezes o número de itens da escala e nunca inferior a 100 indivíduos por análise.

Tendo em consideração o tamanho amostral $(\mathrm{N}=$ 971), utilizado na análise factorial, e o número de itens que constitui a escala, não se colocam problemas de validação.

Inicialmente efectuamos o estudo de consistência interna e como se pode verificar os valores médios oscilaram entre 0,15 no item 14 e os 3,92 no item 2 . Não foram eliminados itens uma vez que todos eles apresentaram um valor correlacional superior a 0,2. Os coeficientes de correlação item total corrigido revelaram que o item 14 foi o que apresentou menor valor correlacional $(r=0,229)$, e menor variabilidade $(17,60 \%)$ não se revelando por isso homogéneo face aos outros itens. A correlação máxima foi obtida no item $2(r=0,762)$, com uma variabilidade de $80,20 \%$.
Quanto aos coeficientes alfa de cronbach estes foram classificados entre bons e muito bons pois variaram entre 0,885 e 0,904, nos mesmos itens

Calculado o índice de fiabilidade pelo método das metades (split-half), obtiveram-se índices mais fracos do que para o alfa global da escala $(0,900)$, sendo para a primeira e segunda metades de 0,865 e 0,800 , respectivamente (cf. Tabela 1 ).

Tabela 1 - Consistência interna da escala de envolvimento face ao álcool em adolescentes

\begin{tabular}{|c|c|c|c|c|c|}
\hline Item & $\bar{x}$ & $\mathrm{Dp}$ & $\begin{array}{l}\text { Correlação } \\
\text { item/total }\end{array}$ & $\mathrm{R}^{2}$ & $\begin{array}{c}\text { Alfa } \\
\text { cronbach } \\
\text { item }\end{array}$ \\
\hline $\begin{array}{l}1 \text { - Com que frequên- } \\
\text { cia costuma tomar } \\
\text { bebidas alcoólicas? }\end{array}$ & 2,65 & 1,46 & 0,717 & 0,741 & 0,888 \\
\hline $\begin{array}{l}2 \text { - Quando tomou o } \\
\text { seu último "copo"? }\end{array}$ & 3,92 & 1,73 & 0,762 & 0,802 & 0,885 \\
\hline $\begin{array}{l}3 \text { - Habitualmente } \\
\text { começo a beber: }\end{array}$ & 1,39 & 0,90 & 0,497 & 0,313 & 0,898 \\
\hline 4 - O que é que bebe? & 2,41 & 1,19 & 0,652 & 0,504 & 0,891 \\
\hline $\begin{array}{l}5 \text { - Como começou a } \\
\text { beber? }\end{array}$ & 2,46 & 1,64 & 0,556 & 0,365 & 0,896 \\
\hline $\begin{array}{l}6 \text { - Quando bebeu pela } \\
\text { primeira vez? }\end{array}$ & 3,35 & 1,44 & 0,694 & 0,581 & 0,889 \\
\hline $\begin{array}{l}7 \text { - A que horas do dia } \\
\text { costuma beber? }\end{array}$ & 1,87 & 1,01 & 0,626 & 0,445 & 0,893 \\
\hline $\begin{array}{l}8 \text { - Da primeira vez } \\
\text { que bebeu porque } \\
\text { razão o fez? }\end{array}$ & 1,53 & 1,13 & 0,505 & 0,283 & 0,897 \\
\hline $\begin{array}{l}9 \text { - Quando bebe, } \\
\text { quanto bebe? }\end{array}$ & 2,23 & 1,48 & 0,712 & 0,555 & 0,888 \\
\hline $\begin{array}{l}10 \text { - Com quem costu- } \\
\text { mas beber? }\end{array}$ & 2,80 & 1,33 & 0,735 & 0,601 & 0,888 \\
\hline $\begin{array}{l}11 \text { - Qual o efeito mais } \\
\text { importante que já teve } \\
\text { com a bebida? }\end{array}$ & 1,79 & 1,50 & 0,645 & 0,496 & 0,891 \\
\hline $\begin{array}{l}12 \text { - Qual a maior con- } \\
\text { sequência da bebida } \\
\text { que já sentiu em toda a } \\
\text { sua vida? }\end{array}$ & 0,93 & 1,61 & 0,464 & 0,369 & 0,900 \\
\hline $\begin{array}{l}13 \text { - Como se sente em } \\
\text { relação ao que bebe? }\end{array}$ & 1,18 & 1,14 & 0,503 & 0,289 & 0,897 \\
\hline $\begin{array}{l}14 \text {-Como o acham os } \\
\text { outros? }\end{array}$ & 0,15 & 0,70 & 0,229 & 0,176 & 0,904 \\
\hline \multirow{2}{*}{ Coeficiente Split-half } & \multicolumn{5}{|c|}{$1^{\circ}$ metade 0,865} \\
\hline & $2^{\mathrm{a}} \mathrm{m}$ & ade 0 & & & \\
\hline $\begin{array}{l}\text { Coeficiente alpha } \\
\text { Cronbach global }\end{array}$ & \multicolumn{5}{|c|}{0,900} \\
\hline
\end{tabular}

Procedeu-se seguidamente ao estudo da análise factorial da escala e o primeiro passo consistiu em analisar o resultado do teste Kaiser-Meyer-Olkin (KMO), o qual afere a qualidade das correlações entre as variáveis. 
Dado o resultado obtido $(\mathrm{KMO}=0,919)$, que foi classificado de excelente, tal como nos refere Marôco (2010) demos continuidade ao estudo factorial da escala. Não foi tido em consideração o teste de Bartlett, uma vez que em grandes amostras, o mesmo leva a rejeitar a hipótese nula.

Ao verificar a proporção da variância de cada variável explicada pelos factores, designada por comunalidade após a extracção, a mesma foi classificada de boa já que oscila entre 0,337 nos itens 3 e 8 e 0,803 no item 2 . Numa análise mais conservadora os itens com comunalidades inferiores a 0.40 deveriam ser eliminados, mas decidimos pela sua manutenção.

Realizada a análise factorial exploratória de componentes principais com os 14 itens, obtiveram-se 2 factores com raízes lactentes superiores a 1 , que no seu conjunto explicaram $55,41 \%$ da variância total (cf. Tabela 2). O teste de scree plot confirmou a retenção de dois factores.

O factor 1, denominado por "hábitos de consumo" explicou $36,90 \%$ da variância total e foi constituído pelos itens $1,2,3,4,5,6,7,8,9$ e 10 .

$\mathrm{O}$ factor 2 foi denominado por "Efeitos, consequências esentimentos face ao beber" explicou $18,51 \%$ da variância total sendo constituído pelos itens 11, 12, 13 e 14 .

Tabela 2 - Ordenação dos itens por factor

\begin{tabular}{|l|c|c|c|}
\hline \multicolumn{1}{|c|}{ Item } & $\begin{array}{c}\text { Factor } \\
1\end{array}$ & $\begin{array}{c}\text { Factor } \\
2\end{array}$ & $\mathrm{H}^{2}$ \\
\hline $\begin{array}{l}\text { 1 - Com que frequência costuma tomar } \\
\text { bebidas alcoólicas? }\end{array}$ & 0,818 & & 0,687 \\
\hline 2 - Quando tomou o seu último "copo"? & 0,889 & & 0,803 \\
\hline 3 - Habitualmente começo a beber: & 0,427 & & 0,337 \\
\hline 4 - O que é que bebe? & 0,747 & & 0,583 \\
\hline 5 - Como começou a beber? & 0,537 & & 0,393 \\
\hline 6 - Quando bebeu pela primeira vez? & 0,807 & & 0,668 \\
\hline 7 - A que horas do dia costuma beber? & 0,702 & & 0,522 \\
\hline $\begin{array}{l}\text { 8 - Da primeira vez que bebeu porque razão } \\
\text { o fez? }\end{array}$ & 0,446 & & 0,337 \\
\hline 9 - Quando bebe, quanto bebe? & 0,615 & & 0,590 \\
\hline 10 - Com quem costumas beber? & 0,775 & & 0,661 \\
\hline $\begin{array}{l}11 \text { - Qual o efeito mais importante que já } \\
\text { teve com a bebida? }\end{array}$ & & 0,616 & 0,585 \\
\hline $\begin{array}{l}12 \text { - Qual a maior consequência da bebida } \\
\text { que já sentiu em toda a sua vida? }\end{array}$ & & 0,755 & 0,601 \\
\hline 13 - Como se sente em relação ao que bebe? & & 0,568 & 0,428 \\
\hline 14 -Como o acham os outros? & & 0,739 & 0,561 \\
\hline
\end{tabular}

\begin{tabular}{|l|c|c|c|}
\hline Factores & Eigenvalue & \% Variância & $\begin{array}{c}\text { \% Variância } \\
\text { Acumulada }\end{array}$ \\
\hline F1 - Hábitos de consumo & 6,291 & 36,90 & 36,90 \\
\hline $\begin{array}{l}\text { F2 - Efeitos, consequências } \\
\text { e sentimentos face ao beber }\end{array}$ & 1,466 & 18,51 & 55,41 \\
\hline
\end{tabular}

Ainda no que diz respeito aos estudos de validade, foram determinados os valores de alfa de cronbach para cada um dos factor. Como se pode verificar pela tabela 3, o primeiro factor revelou uma boa consistência interna com um alfa de cronbach de 0,899). Já com o segundo encontrou-se uma razoável consistência interna ao apresentar um alfa de 0,689 . O valor obtido na escala global $(0,900)$ revelou-se superior ao valor obtido na escala do estudo original $(0,887)$ de Fonte \& Alves (1999).

Tabela 3 - Comparação dos valores de alfa de cronbach do estudo actual com a escala original por factores

\begin{tabular}{|l|l|l|l|l|c|}
\hline \multirow{2}{*}{ Factores } & \multirow{2}{*}{$\begin{array}{l}N^{\text {I }} \\
\text { Itens }\end{array}$} & \multicolumn{4}{|c|}{ Alfa de Cronbach } \\
\cline { 3 - 6 } & & \multicolumn{2}{|c|}{ Split-half } & \multicolumn{2}{|c|}{ Total } \\
\cline { 3 - 6 } & $\begin{array}{l}1^{\text {a }} \\
\text { Metade }\end{array}$ & $\begin{array}{l}2^{\text {a }} \\
\text { Metade }\end{array}$ & $\begin{array}{l}\text { Estudo } \\
\text { actual }\end{array}$ & $\begin{array}{l}\text { Estudo } \\
\text { original }\end{array}$ \\
\hline $\begin{array}{l}\text { Factor 1 - Hábi- } \\
\text { tos de consumo }\end{array}$ & 10 & 0,807 & 0,812 & 0,899 & - \\
\hline $\begin{array}{l}\text { Factor 2 - Efei- } \\
\text { tos, consequên- } \\
\text { cias e sentimen- } \\
\text { tos face ao beber }\end{array}$ & 4 & 0,692 & 0,377 & 0,689 & - \\
\hline AAIS (Global) & 14 & 0,865 & 0,800 & 0,900 & 0,887 \\
\hline
\end{tabular}

Uma contribuição para o estudo da validade da escala é a determinação da matriz de correlação entre os diversos factores e o valor global da escala. Vaz Serra (1994) cit. por Duarte (2008) refere que as correlações não devem ser demasiado elevadas, uma vez que este facto indica-nos que os itens são redundantes, sendo preferível existir uma correlação moderada entre si, facto que nos revela uma sensibilidade a aspectos diferentes do mesmo constructo.

As correlações entre os dois factores e com a pontuação global da escala variaram entre 0,582 (F1 vs F2) e 0,970 (F1 vs AAIS Global), sendo altamente significativos. Entre os factores em análise, bem como com a pontuação global da escala verificou-se a existência de correlações positivas, significando que ao aumento ou diminuição da pontuação num dos factores se encontra igualmente associado a um aumento ou diminuição de pontuação no fator com o qual se associa.

Tabela 4 - Matriz de correlação de Pearson entre os factores e a pontuação global da AAIS

\begin{tabular}{|l|c|c|}
\hline Factores & Factor 1 & Factor 2 \\
\hline Factor 2 & $0,582^{\star \star}$ & - \\
\hline AAIS (Global) & $0,970^{\star \star}$ & $0,763^{\star \star}$ \\
\hline
\end{tabular}

${ }^{* *} \mathrm{p}<0,01$ 


\section{DISCUSSÃO}

A Escala de Envolvimento com o Álcool para Adolescentes é um questionário de auto-avaliação constituído por 14 questões, dirigido especificamente para populações jovens, com o objectivo de quantificar o grau de relacionamento destes com o uso do álcool. A análise factorial de componentes principais, com rotação ortogonal de tipo varimax. Obtiveram-se 2 factores com raízes lactentes superiores a 1 , que no seu conjunto explicaram $55,41 \%$ da variância total. $\mathrm{O}$ factor 1 foi denominado por "hábitos de consumo" explicou $36,90 \%$ da variância total, e o factor 2 foi denominado por "Efeitos, consequências e sentimentos face ao beber" e explicou $18,51 \%$ da variância total. Estes resultados diferem dos valores obtidos por Fonte \& Alves (1999), os quais obtiveram uma estrutura factorial a 3 factores responsáveis por $58 \%$ da variância total.

O Alfa de Cronbach para a globalidade da escala foi de $\alpha=0.900$. Obteve Split-half da Primeira metade $=$ 0,865 , da Segunda metade $=0,800$ Coeficiente Splithalf da Primeira metade $=0,865$, Segunda metade $=$ 0,800 . Segunda metade $=0,800$ Valores considerados de referencia, Marôco (2010).

\section{CONCLUSÃO}

A Escala de Envolvimento com o Álcool para Adolescentes (AAIS), desenvolvido por Mayer e Filstead Foi validado para a população adolescente portuguesa por Fonte \& Alves (1999). A Revalidação revela boas propriedades psicométricas, indicando que poderá ser utilizada em ensaios futuros no intuito de dar a conhecer os comportamentos de consumo de álcool nesta facha etária.

\section{IMPLICAÇÕES PARA A PRÁTICA CLÍNICA}

A qualidade psicómetrica da Escala de Envolvimento com o Álcool para Adolescentes (AAIS) permite a sua utilização enquanto ferramenta de trabalho nas atividades de promoção de estilos de vida saudáveis dos adolescentes.

\section{REFERÊNCIAS BIBLIOGRÁFICAS}

Barros, L. M. D. (2003). O envolvimento da criança de três anos em grupos heterogéneos: Um estudo em contextos pedagógicos diferenciados. Dissertação de mestrado não publicada, Universidade do Minho, Braga.

Duarte, J. C. (2008). Privação do sono, rendimento escolar e equilíbrio psico-afectivo na adolescência. Dissertação de doutoramento não publicada, Instituto de Ciências Biomédicas Abel Salazar, Universidade do Porto, Porto.

Fonte, A., \& Alves, A., (1999). Uso da Escala de Envolvimento com o Álcool para Adolescentes (AAIS). Avaliação das Características Psicométricas. Alcoologia, Revista da Sociedade Portuguesa de Alcoologia, VII (4), s.p. Acedido em 12, maio, 2011, em http://anibalfonte.home.sapo.pt/AAIS.html.

Mayer, J. E. \& Filstead, W.J., (1979). The Adolescent Alcohol Involvement Scale: an instrument for measuring adolescent use and misuse of Alcohol. Study. Journal of Studies on Alcohol and Drugs, 40(3), 291-300.

Marôco, J., (2010). Análise estatística com o PASW Statistics. Pêro Pinheiro: Report Number, Lda.

Mello, M. L. M., Barrias, J., \& Breda, J., (2001). Álcool e problemas ligados ao álcool em Portugal. Lisboa: Direcção-Geral da Saúde.

Pestana, M. H., \& Gageiro, J. N., (2008). Análise de Dados para Ciências Sociais: A Complementaridade do SPSS ( $5^{a}$ ed., revista e corrigida). Lisboa: Edições Sílabo.

Reis, A., Barros, J., Fonseca, C., Parreira, L., Gomes, M., Figueiredo, I., \& Matapa, S., (2011). Prevalência da Ingestão de Álcool nos Adolescentes - Estudo PINGA. Revista Portuguesa de Clínica Geral, 27, 338 - 346. 


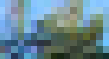

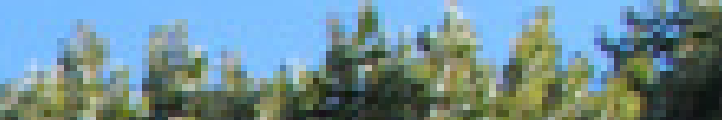

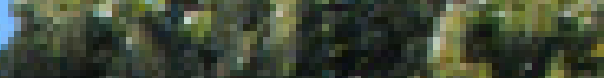

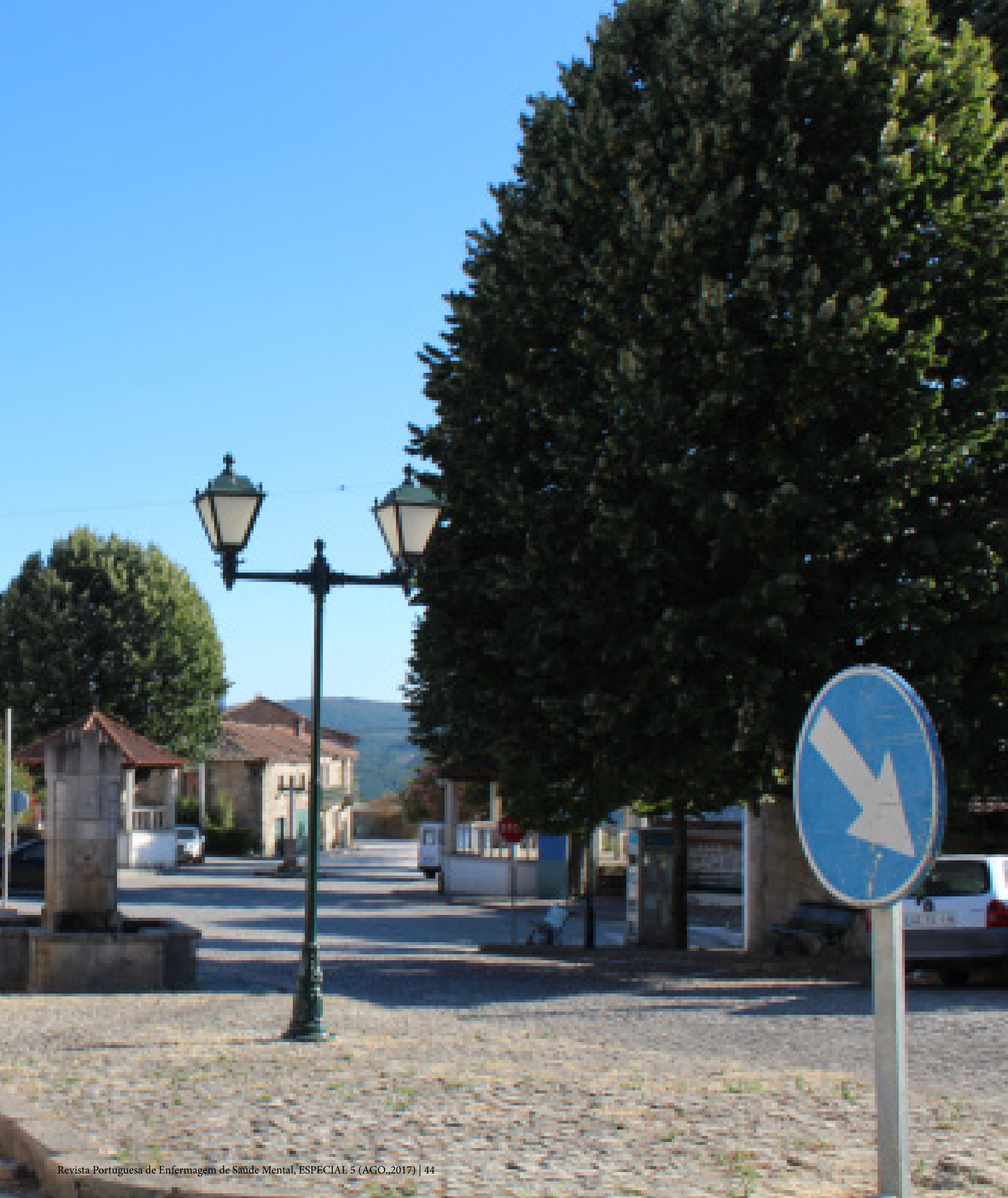

